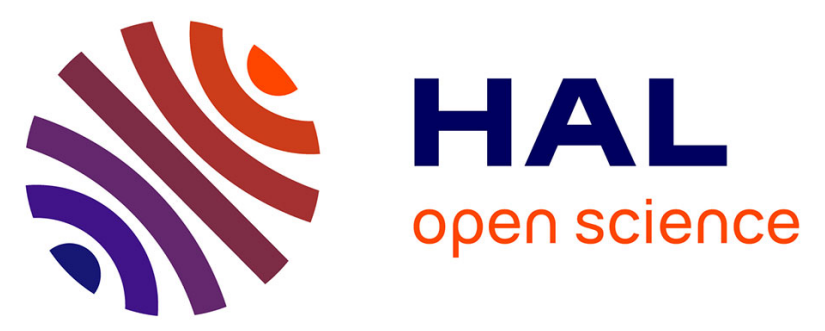

\title{
The effect of cross-contamination in the sequential interfacial polymerization on the RO performance of polyamide bilayer membranes
}

Young-Hye La, Jacquana Diep, Radwan Al-Rasheed, Majed Nassar, E Idil Mouhoumed, Anthony Szymczyk, Geraud Dubois

\section{To cite this version:}

Young-Hye La, Jacquana Diep, Radwan Al-Rasheed, Majed Nassar, E Idil Mouhoumed, et al.. The effect of cross-contamination in the sequential interfacial polymerization on the RO performance of polyamide bilayer membranes. Journal of Membrane Science, 2014, 466, pp.348 - 356. 10.1016/j.memsci.2014.05.005 . hal-01077845

HAL Id: hal-01077845

https://hal-univ-rennes1.archives-ouvertes.fr/hal-01077845

Submitted on 27 Oct 2014

HAL is a multi-disciplinary open access archive for the deposit and dissemination of scientific research documents, whether they are published or not. The documents may come from teaching and research institutions in France or abroad, or from public or private research centers.
L'archive ouverte pluridisciplinaire HAL, est destinée au dépôt et à la diffusion de documents scientifiques de niveau recherche, publiés ou non, émanant des établissements d'enseignement et de recherche français ou étrangers, des laboratoires publics ou privés. 


\title{
The Effect of Cross-contamination in the Sequential Interfacial Polymerization on the RO Performance of Polyamide Bilayer Membranes
}

Young-Hye La, ${ }^{1 *}$ Jacquana Diep, ${ }^{1}$ Radwan Al-Rasheed, ${ }^{2}$ Majed Nassar, ${ }^{2}$ E. Idil Mouhoumed ${ }^{3}$, Anthony Szymczyk, ${ }^{3}$ Geraud Dubois ${ }^{1 *}$

${ }^{1}$ IBM Almaden Research Center, 650 Harry Road, San Jose, CA 95120

${ }^{2}$ King Abdulaziz City for Science and Technology (KACST), Kingdom of Saudi Arabia, P.O Box 6086, Riyadh 11442.

${ }^{3}$ Université de Rennes 1, Institut des Sciences Chimiques de Rennes (UMR CNRS 6226), 263 Avenue du Général Leclerc, 35042 Rennes, France

\author{
*Corresponding authors: Dr. Young-Hye La, Tel: +1 408927 1256; Fax: +1 4089273310 \\ E-mail address: yna@us.ibm.com \\ Dr. Geraud Dubois, Tel: +1 408927 1584; E-mail address: gdubois@us.ibm.com
}

\begin{abstract}
In this study, hexafluoroalcohol-containing polyamide layer (HFAPA) was prepared on top of a conventional polyamide under-layer (REFPA) via sequential interfacial polymerization (SIP) to improve RO separation behavior, and the performance of the resulting bilayer membrane was thoroughly optimized by investigating the effect of cross-contamination in the SIP process. When several coupons of the polyamide bilayer membrane were prepared by SIP of $\mathrm{MPD}_{(\mathrm{aq})}, \mathrm{TMC}_{(\mathrm{hx})}$ and hexafluoroalcohol-containing
\end{abstract}


diamine (HFAMDA) $)_{\text {(aq) }}$ in the manner of subsequent membrane dipping, unreacted MPD monomer (mostly captured in the porous PSF support) carried over from the $1^{\text {st }}$ interfacial reaction dissolved and accumulated in the $2^{\text {nd }}$ aqueous solution as verified by UV spectroscopic analysis. The MPD contaminant then participated in the $2^{\text {nd }}$ interfacial reaction, forming copolyamide with HFAMDA monomer onto the REFPA. Depending on the amount of MPD contaminant accumulated in the $2^{\text {nd }}$ aqueous solution, the composition of the resulting co-polyamide in the top-layer varied, causing a significant variation of RO performance; the flux was gradually decreased with the increase of MPD contaminants while the salt rejection slightly increased (from $1^{\text {st }}$ coupon toward $4^{\text {th }}$ coupon). This result indicated that a trace amount of MPD contaminant may be necessary to maximize RO separation behavior. Through in-depth performance evaluation of polyamide bilayer membranes prepared by adding various known-amount of MPD into $2^{\text {nd }}$ HFAMDA solution, and also by applying a frame process $\left(2^{\text {nd }}\right.$ amine solution was applied only top surface of membrane) to eliminate uncontrollable MPD contamination, we have successfully demonstrated consistent RO performance, and identified an optimum material composition to provide superior separation performance. The bilayer membrane prepared by adding $1.2 \mathrm{~mol} \%$ of MPD to the total amount of HFAMDA in the $2^{\text {nd }}$ aqueous solution showed $99.8 \% \mathrm{NaCl}$ rejection with the water flux of $45 \mathrm{LMH}$ under the cross-flow filtration performed with $2000 \mathrm{ppm} \mathrm{NaCl}$ solution at $400 \mathrm{psi}, 25^{\circ} \mathrm{C}$.

\section{Keywords}

Reverse Osmosis, Polyamide Bi-layer Membrane, Cross-contamination, Sequential Interfacial Polymerization (SIP), Desalination. 


\section{Introduction}

Membrane technology, particularly reverse osmosis (RO) is widely used for water desalination and water reuse to overcome global water scarcity. High performance membrane materials and processes for these applications have attracted immense interest from the scientific community [1-2]. The current generation of RO membranes is based on the thin film composite (TFC) structure, where an ultra-thin $(50 \sim 200 \mathrm{~nm})$ active layer exists on a thick porous support [3-5]. The ultra-thin active layer mainly determines the membrane's separation performance while the support (porous polysulfone and a non-woven polyester fabric) provides enough mechanical strength for high operation pressure. To date, various materials such as polyamide [6-10], poly(ether-amide) [11], polyurea [12-13], poly(amide-urethane) [10-11], and sulfonated poly(arylene ether sulfone) [14] have been investigated as potential active layers [15]. However, the crosslinked aromatic polyamide invented by Cadotte et al. in the 1980s still appears as the most reliable material showing superior salt rejection and water flux, and remains as a basic platform for manufacturing TFC RO membranes on a commercial scale [7].

The TFC membrane comprising aromatic polyamide active layers often suffer from membrane fouling [16] and chemical oxidation by chlorine-based biocidal agents [17]. Therefore, recent research has focused on surface modification of the crosslinked polyamide TFC membrane to remedy its weakness or to further improve its separation performance. Self-assembly of inorganic nanoparticles (ex. $\mathrm{TiO}_{2}$ ) or organic functional materials (ex. poly(ethylene amine)) onto commercial polyamide membranes through hydrogen-bond or electrostatic interaction has been introduced as a way to improve a membrane's anti-fouling efficiency and/or chlorine resistance [18-20]. Surface-initiated 
grafting of anti-fouling or chlorine resistant materials (ex. polyethyleneglycol methacrylate, imidazolidinyl urea) has been also performed by activating a polyamide membrane surface with an appropriate coupling agent or a redox initiator [21-22]. Contrary to these strategies, in which a commercial TFC polyamide membrane with residual carboxylate functional groups $\left(\mathrm{COO}^{-}\right)$has been used as a base material, direct modification of a freshly prepared polyamide layer by a sequential interfacial reaction scheme has been also proposed [23-25]. In general, a crosslinked polyamide layer is prepared by interfacial polymerization of m-phenylenediamine (MPD) in water and trimesoylchloride (TMC) in an organic solvent. Thus, the membrane surface always contains excessive unreacted acid chlorides (-COCl), which will eventually hydrolyze to carboxylic acids $(\mathrm{COOH})$ as normally observed on the surface of conventional aromatic polyamide RO membranes. The hydrolysis reaction, however, is relatively slow, so the acid chlorides are available for further interfacial reactions with the same or different types of amine-functionalized monomers before hydrolysis occurs [24-25]. This sequential interfacial polymerization is very straightforward and can be done in a short period of time since the reaction between amine and acid chlorides occurs almost instantly if monomers have good mobility [23, 25]. Kang et al. immobilized aminefunctionalized polyethylene glycol $\left(\mathrm{NH}_{2}-\mathrm{PEG}\right)$ onto a conventional aromatic polyamide active layer by using the sequential interfacial reaction scheme and demonstrated improved anti-fouling efficiency [24]. Zou et al. also fabricated an anti-fouling polyamide layer with a large amount of amino groups on the surface by dipping a conventional polyamide made with $\mathrm{MPD}_{(\mathrm{aq})}$ and $\mathrm{TMC}_{(\mathrm{hx})}$ into MPD aqueous solution again [25]. Similarly, we have developed polyamide bilayer membranes (HFAPA-on- 
REFPA) composed of an hexafluoroalcohol (HFA)-containing polyamide top-layer (HFAPA) and a conventional polyamide underlayer (REFPA) with enhanced RO separation performance by using the sequential interfacial polymerization process of $\mathrm{MPD}_{(\mathrm{aq})}, \mathrm{TMC}_{(\mathrm{hx})}$, and hexafluoroalcohol-containing diamine (HFAMDA) [23].

Although the sequential interfacial reaction or polymerization (SIP) enables the formation of a very thin and durable coating (safe from delamination) on a conventional polyamide membrane, each process step must be well controlled to obtain reliable and consistent membrane performance. One key feature of SIP dipping process that we have identified in this study is cross-contamination of water-soluble diamine monomers. As shown in Figure 1, to fabricate a performance enhancing layer on a conventional polyamide (REFPA) via the SIP process, a roll or flat-sheets of REFPA membrane prepared by the interfacial reaction of MPD in the first aqueous solution (I) and TMC in an organic solution will continuously pass through the second aqueous solution (II) containing another amine functional monomers (ex. HFAMDA) in serial order. In this sequential process, the contamination of the second aqueous solution by the water soluble-, unreacted MPD monomers, which are transferred from the previous reaction step, could be a significant issue, causing a huge variation in the material compositions and $\mathrm{RO}$ performance of the resulting polyamide membranes; $\mathrm{RO}$ performance at the end of the roll (or the last sheet) could be very different from that at the starting roll (or the first sheet) due to the accumulation of MPD contaminant in the $2^{\text {nd }}$ amine solution. This cross-contamination can be minimized or eliminated by applying reaction solutions onto only top surface of membranes by using a spray or other equivalent methods since most of the MPD monomers are delivered by the thick porous support layer during a 
membrane dipping process. However, an optimum amount of MPD involvement in the $2^{\text {nd }}$ interfacial reaction along with a base diamine monomer (HFAMDA) may provide positive influence on the membrane performance, and it is therefore very valuable to investigate the effect of MPD cross-contamination on the material compositions of polyamide membranes and their final RO performance in a systematic way.

Here, we have prepared multiple coupons of polyamide bilayer membranes (HFAPA-on-REFPA) via sequential interfacial polymerization of $\mathrm{MPD}_{(\mathrm{aq})}, \mathrm{TMC}_{(\mathrm{hx})}$ and $\operatorname{HFAMDA}_{(\mathrm{aq})}$ in the manners of serial membrane dipping and investigated the effect of MPD contamination on the material composition as well as desalination performance of the resulting polyamide membranes. Quantitative analysis of the MPD leaching and its reactivity in the $2^{\text {nd }}$ aqueous solution was confirmed by UV spectroscopic study, and the relative material compositions of polyamide bilayer membranes were characterized by Xray photoelectron spectroscopy (XPS). RO desalination performance evaluated by using cross-flow filtration of saline feed water demonstrated that a trace amount of MPD is necessary in the $2^{\text {nd }}$ diamine solution $\left(\operatorname{HFAMDA}_{(\mathrm{aq})}\right)$ to optimize overall desalination performance of the polyamide bilayer membranes. The ideal composition between MPD and HFAMDA to form the best performing HFAPA top-layer was also identified through an in-depth study of the performance of copolyamide top-layers, which were prepared by adding various known-amounts of MPD into the $2^{\text {nd }}$ HFAMDA solution and also by applying a frame process ( $2^{\text {nd }}$ amine solution was applied only top surface of membrane) to eliminate uncontrollable MPD contamination. 


\section{Experimental}

\subsection{Materials}

3,3'-bis(1-hydroxy-1-trifluoromethyl-2,2,2-trifluoroethyl)-4,4'-methylenedianiline (HFA-MDA) was provided by Central Glass, Inc. (Japan) and used as received. m-phenylenediamine (MPD flakes, > $99 \%$ ) and trimesoyl chloride (TMC, $98 \%$ ) were purchased from Sigma-Aldrich. MPD was purified by sublimation, and TMC was distilled before use. Sodium chloride $(\mathrm{NaCl})$, sodium hydroxide $(\mathrm{NaOH}$, pellets) and sodium carbonate $\left(\mathrm{Na}_{2} \mathrm{CO}_{3}\right)$ were purchased from J. T. Baker. Hexane was purchased from OmniSolv. Deionized (DI) water was used for all experiments. Polysulfone (PSF) ultrafiltration membranes (PS20) were purchased from Sepro Membranes, Inc. and used as supports for RO fabrication.

\subsection{Fabrication of TFC Polyamide Membranes}

Membrane fabrication was performed by either a dipping process or a frame process. The dipping process required a polysulfone (PSF) ultrafitration membrane to be submerged in solution and held into place by two glass plates at opposite ends. The frame process required the membrane to be clamped between two frames and only the top surface of the membrane was exposed to the reaction solution, allowing for a one-sided interfacial polymerization. Where the frame process was not mentioned, the dipping process was used for the membrane fabrication process.

Reference polyamide TFC membrane (REFPA): A PSF UF membrane was placed in an aqueous solution of $2 \%(\mathrm{w} / \mathrm{v}) \mathrm{MPD}$ for $2 \mathrm{~min}$, and the membrane was rolled with a rubber roller to remove excess solution. The MPD-saturated PSF membrane was then 
immersed in a solution of $0.1 \%(\mathrm{w} / \mathrm{v}) \mathrm{TMC}$ in hexane. After $1 \mathrm{~min}$, the TMC solution was decanted, and the resulting membrane was rinsed with an aqueous solution of $0.2 \%$ (w/v) sodium carbonate and stored in DI water until use.

Polyamide Bilayer TFC Membranes (HFAPA-on-REFPA) with same solution bath (for cross-contamination study): A polyamide bilayer membrane, HFAPA-on-REFPA, was synthesized on a pre-formed polysulfone (PSF) ultrafiltration membrane by sequential interfacial polymerization. The PSF membrane was placed in an aqueous solution of $2 \%$ (w/v) MPD for $2 \mathrm{~min}$, and the MPD soaked support membrane was then rolled with a rubber roller to remove excess solution. The MPD saturated membrane was then immersed in a solution of $0.1 \%(\mathrm{w} / \mathrm{v}) \mathrm{TMC}$ in hexane. After $1 \mathrm{~min}$, the resulting membrane was air dried at ambient temperature and placed in an alkaline aqueous solution containing $2 \%(\mathrm{w} / \mathrm{v}) \mathrm{HFA}-\mathrm{MDA}$ diamine for 2 min $(2$ eq. of $\mathrm{NaOH}$ per mole of HFA-MDA were added to completely dissolve the HFA-MDA monomer in water). The resulting membrane was subsequently rinsed with an aqueous $0.2 \%(\mathrm{w} / \mathrm{v})$ sodium carbonate solution for $5 \mathrm{~min}$, and stored in DI water until use. To investigate the effect of MPD cross-contamination on the final RO performance, 4 coupons (4" $\mathrm{x} 5$ ") of the polyamide bilayer membrane were prepared by using the same reaction solutions $\left(\mathrm{MPD}_{(\mathrm{aq})}, \mathrm{TMC}_{(\mathrm{hx})}\right.$, and $\left.\operatorname{HFAMDA}_{(\mathrm{aq})}\right)$.

Polyamide Bilayer TFC Membranes (HFAPA-on-REFPA) by a frame process (Copolymerization of $2^{\text {nd }}$ layer): REFPA was first prepared by the dipping process. Then, copolyamide top-layers were synthesized by exposing the top surface of REFPA 
membranes to the $2^{\text {nd }}$ aqueous solutions by using a frame process. The $2^{\text {nd }}$ aqueous solutions to form copolyamide top-layers were formulated by adding various amounts of MPD additives ( $1 \mathrm{~mol} \% \sim 5 \mathrm{~mol} \%$ relative to HFAMDA) into $2 \mathrm{wt} \%$ HFAMDA base solution.

\subsection{Characterization of TFC Membranes}

X-ray Photoelectron Spectroscopic (XPS) analysis: XPS analysis was performed using a Physical Electronics Quantum 2000 ESCA Microprobe with a monochromatic Al Ka source and charge neutralization. $1100 \mathrm{eV}$ survey spectra were taken at $188 \mathrm{eV}$ pass energy, $200 \mu \mathrm{m}$ spot size, and $45^{\circ}$ take-off angle for each sample to determine the overall elemental composition of the membrane's active layer surface. High resolution spectra (58 eV pass energy, $0.5 \mathrm{eV} / \mathrm{step})$ were taken for carbon (1s), oxygen (1s), nitrogen (1s), and fluorine (1s) to investigate the chemical environment. Binding energies were referenced to $\mathrm{C}(1 \mathrm{~s})$ maximum $=284.8 \mathrm{eV}$.

Atomic force microscopic (AFM) analysis: Membrane surface topographic images were acquired by AFM using a Digital Instruments Dimension 3100 atomic force microscope with a Nanoscope IV controller (Woodbury, NY). Samples were dried under a vacuum prior to analysis and the AFM images were acquired under ambient conditions in intermittent contact mode (tapping mode) at a $1 \mathrm{~Hz}$ scan rate and $256 \times 256$ pixel resolution with silicon cantilevers (spring constant: $\sim 50 \mathrm{~N} / \mathrm{m}$ ). Three different positions were analyzed for each sample, each over a $5 \mu \mathrm{m} \times 5 \mu \mathrm{m}$ area. Surface roughness was calculated using the data analysis software provided by the manufacturer. 
Transmission electron microscopic (TEM) analysis: Formation of thin, dense, interfacially polymerized polyamide membranes was confirmed by cross-sectional TEM. A membrane sample was dried and cut into $5 \mathrm{~mm} \times 5 \mathrm{~mm}$ pieces. The non-woven fabric backing layer (of the UF support membrane) was peeled away before embedding the sample in Epotek 301 epoxy and outgassing in a vacuum chamber for 1 hour. The sample pieces were cured in a $60{ }^{\circ} \mathrm{C}$ oven for 3 hours. The samples were then prepared for TEM imaging by cutting thin sections using a Leica Ultracut S Ultramicrotome and collecting the pieces on copper grids. All samples were imaged at $200 \mathrm{kV}$ in a Topcon 002B TEM.

Fourier Transform Infrared Sepectroscopic (FTIR) analysis: FTIR spectra of membranes were obtained by using Thermo Nicolet Nexus 670 FT-IR with Smart Omni Sampler designed for attenuated total reflectance infrared spectroscopy (ATR-IR). All membrane coupons were thoroughly dried under vacuum at room temperature for at least 24 hours before recording the measurements. The IR detector (MCT) was cooled with liquid nitrogen for 30 minutes before using and 128 scans for each sample were recorded with the resolution of $4 \mathrm{~cm}^{-1}$.

Contact angle measurement: Water contact angles of the TFC membranes were measured by a static sessile drop method using a Dataphysics Contact Angle System OCA 20 (Germany). All samples were dried under vacuum prior to analysis. 
Streaming current measurement: Streaming current measurements were performed with a SurPASS electrokinetic analyzer (Anton Paar GmbH). All measurements were conducted with an adjustable-gap cell which makes possible to vary the distance between the two membrane samples with micrometric screws without dismounting the cell. The membrane was cut and adjusted to the dimensions of the sample holders (i.e. $L=2 \mathrm{~cm}$ and $W=1 \mathrm{~cm})$ and fixed using double-sided adhesive tape. To prevent any leakage between the membranes and the sample holders, membranes were firmly pressed against sample holders for 30-60 s, paying special attention to the edges exposed to the hydrodynamic flow. The solution flow was created by a pair of syringe pumps and streaming current was measured with a pair of reversible $\mathrm{Ag} / \mathrm{AgCl}$ electrodes (surface area: $10 \mathrm{~cm}^{2}$ ).

UV analysis: The amounts of MPD monomers transferred from previous reaction bath were measured using Agilent 8453 UV-Visible Spectrophotometer. The amounts were estimated using the calibration curve generated from known MPD concentrations. Five molar concentrations, $0.0000462 \mathrm{M}, 0.0000925 \mathrm{M}, 0.0001849 \mathrm{M}, 0.0002774 \mathrm{M}$, and $0.0004624 \mathrm{M}$, were used to generate the calibration curve using linear regression to fit the following equation:

$$
\mathrm{A}=\varepsilon^{*} \mathrm{C} * \mathrm{~L}
$$

Where $\mathrm{A}$ is the absorbance, $\varepsilon$ is the constant known as extinction coefficient $\left(\mathrm{M}^{-1} \mathrm{~cm}^{-1}\right)$, $\mathrm{C}$ is the molar concentration (M) of the MPD, and $\mathrm{L}$ is the pathlength through the sample. 
A quartz cuvette with a pathlength, $\mathrm{L}$, of $1 \mathrm{~cm}$ was used for the analysis. An $\varepsilon$ value of $2281.3 \mathrm{M}^{-1} \mathrm{~cm}^{-1}$ was obtained from the linear regression with an $\mathrm{R}^{2}$ of 0.9996 .

\subsection{Evaluation of Separation Performance}

$\mathrm{NaCl}$ rejection and water permeance measurement: Membrane performance was evaluated using a cross-flow filtration system. Membrane samples were clamped into the cross-flow cells, and $25{ }^{\circ} \mathrm{C}$ DI water was circulated throughout the feed loop at 27.6 bar (400 psi) and a cross-flow rate of $3.8 \mathrm{~L} \mathrm{~min}^{-1}$ for 3 hours to allow the membranes to equilibrate and reach steady state. After reaching steady state, the pure water flux was measured gravimetrically. Subsequently, $2000 \mathrm{ppm} \mathrm{NaCl}$ was added to the feed, and after allowing the system to reach steady state, the conductivities of the feed and permeate solutions were measured and converted to salt concentration via a calibration curve. The apparent salt rejection, $\mathrm{R}$, was calculated as follows:

$\mathrm{R}(\%)=100 \times\left(1-\left(\mathrm{C}_{\mathrm{p}} / \mathrm{C}_{\mathrm{f}}\right)\right)$,

where $C_{p}$ and $C_{f}$ are the salt concentrations of the permeate and feed solutions, respectively.

Water flux was expressed as permeate volume per membrane area per unit of time $\left(\mathrm{L} \mathrm{m}^{-2}\right.$ $\left.\mathrm{h}^{-1}: \mathrm{LMH}\right)$. 


\section{Results and Discussion}

\section{3-1. Polyamide Bilayer Membranes: The Effect of Cross-contamination on RO Performance}

As described in the Introduction, cross-contamination during sequential interfacial polymerization (SIP) might be a critical issue causing large variations in membrane performance. In order to investigate the effect of MPD contamination in the $2^{\text {nd }}$ aqueous solution on the RO performance of the resulting membranes, polyamide bilayer membranes (HFAPA-on-REFPA) composed of an aromatic polyamide under-layer (REFPA) and a HFA-substituted aromatic polyamide top-layer (HFAPA) were fabricated by sequential interfacial polymerization of $\mathrm{MPD}_{(\mathrm{aq})}, \mathrm{TMC}_{(\mathrm{hx})}$, and $\operatorname{HFAMDA}_{(\mathrm{aq})}$ in the manner of sequential membrane dipping, and 4 sample coupons were fabricated with the same reaction solutions in serial order as shown in Figure 1. For comparison, TFC membranes with a single layered conventional aromatic polyamide layers (REFPA) were also prepared by one-step interfacial polymerization of MPD and TMC. The formation of thin and dense polyamide active layers was confirmed by cross-sectional TEM images, however it was difficult to identify a difference in the active layer thickness between REFPA and HFAPA-on-REFPA membranes as we demonstrated in our earlier paper (active layer thickness: approximately $80 \sim 85 \mathrm{~nm}$ dense film with $250 \sim 300 \mathrm{~nm}$ overall thickness including extended branch-like arms, Supporting Information, Figure S1) [23].

Figure 2(a) and 2(b) shows water flux and salt rejection of four HFAPA-on-REFPA membrane coupons evaluated by using cross-flow filtration with a $2000 \mathrm{ppm} \mathrm{NaCl}$ feed solution. As we expected, the desalination performance of the resulting polyamide membranes was influenced by membrane fabrication order. The first sample coupon 
always exhibited the highest water flux (ca. $57 \mathrm{LMH}$ ), and the flux gradually decreased towards the last $\left(4^{\text {th }}\right)$ sample coupon, while salt rejection slightly increased from the $1^{\text {st }}$ coupon $(99.4 \%)$ to the $4^{\text {th }}$ coupon $(99.6 \%)$. When compared with desalination performance of REFPA (water flux: $57 \pm 7 \mathrm{LMH}$, salt rejection: $98.9 \pm 0.5$ ), all four samples showed much higher salt rejection with similar or lower water flux (1st sample coupon showed similar water flux to REFPA). However, this trend was not observed when we prepared four coupons of REFPA by dipping into the same $\operatorname{MPD}_{(\mathrm{aq})}$ and $\mathrm{TMC}_{(\mathrm{hx})}$ solutions in serial order, which indicates that the observed performance trend (flux reduction and rejection increase from $1^{\text {st }}$ to $4^{\text {th }}$ coupons) from the polyamide bilayer membranes are solely generated in the $2^{\text {nd }}$ reaction solution. The most plausible mechanism to explain this phenomenon is that, as we initially expected, the unreacted water-soluble monomers (MPD) carried over from the first reaction step dissolve and accumulate in the second aqueous solution, and ultimately participate in the second interfacial reaction with HFAMDA monomers to form a much denser copolyamide structure as a top-layer. Copolymer-formation on the top-surface was also confirmed by XPS and contact angle analysis. As shown in Table 1, the surface fluorine signal $(\mathrm{F}(1 \mathrm{~s}))$ and an atomic ratio of fluorine to carbon $(\mathrm{F} / \mathrm{C})$ decreased from $1^{\text {st }}$ coupon towards $4^{\text {th }}$ coupon. This result supports that MPD incorporation to the top HFAPA layer gradually increases as the amount of MPD contaminant in the $2^{\text {nd }}$ HFAMDA solution increases. The slight reduction of surface water contact angles depending on the membrane fabrication order $\left(133^{\circ}\right.$ for the $1^{\text {st }}$ coupon to $120^{\circ}$ for the $\left.4^{\text {th }}\right)$ also indicates that the relative content of hydrophobic HFA groups decreases due to more MPD involvement in the $2^{\text {nd }}$ interfacial reaction. 


\section{3-2. Quantitative Analysis of MPD Leaching}

The MPD contamination issue and the performance trend we observed from polyamide bilayer membranes (HFAPA-on-REFPA) brought up the question about actual compositions of the bilayer membranes and even the REFPA membranes we have prepared as our internal control. In order to clarify material compositions, MPD leaching and its involvement in the $2^{\text {nd }}$ interfacial reaction were quantitatively monitored by using UV-visible spectrophotometer through two case studies as shown in Figure 3. For Case 1 (Figure 3(a)), four coupons of REFPA membrane were prepared by the single interfacial reaction of $\mathrm{MPD}_{(\mathrm{aq})}$ and $\mathrm{TMC}_{(\mathrm{hx})}$, and the membrane coupons were directly immersed into fresh deionized water ( 2 min- immersion for each sample coupon) in serial order. Each coupon had a fixed dimension of 4" x 5" for quantitative analysis. In general, a PSF support will capture lots of MPD within its pores (MPD aqueous solution fills up the pores of PSF support) as well as its surface when it was initially soaked to a $\operatorname{MPD}_{(\mathrm{aq})}$ solution. By dipping the MPD-saturated PSF support into a $\mathrm{TMC}_{(\mathrm{hx})}$ solution, the MPD molecules on the membrane surface react with TMC, but the MPD molecules in the pores still remains without leaching in the TMC-organic solvent. As a result, the REFPA membrane prepared by the $1^{\text {st }}$ interfacial reaction typically has large amounts of unreacted MPD in the support layer and unreacted TMC deposited on its surface. When this REFPA membrane was immersed into deionized water, water soluble MPD monomers leaches out, and consequently some of them could directly react with the surface-remaining unreacted TMC monomers $\left(2^{\text {nd }}\right.$ interfacial reaction). If so, the UV signals of the water solution would be originated from only the amount of the leftover- 
MPDs from the $2^{\text {nd }}$ interfacial reaction. To confirm this in-situ $2^{\text {nd }}$ interfacial reaction between the released-MPD and the surface residual TMC molecules, Case Study 2 was also performed as shown in Figure 3(b). In the Case 2, unreacted free TMC monomers deposited on the REFPA was thoroughly rinsed with hexane before dipping into deionized water. Since the REFPA surface also has reactive acid chloride groups, which are chemically bound to the polyamide layer, very small portion of MPD released in the water could still be consumed by some of these surface-anchored acid chlorides. But, MPD amounts detected by the UV intensities of the water solution would be much closer to the actual amount of MPD released from membrane coupons in this case, compared to the Case Study 1.

Figure 4(a) and 4(b) shows the UV intensities of aqueous rinse solutions from the case studies 1 and 2, respectively. In both cases, strong absorption peaks at $290 \mathrm{~nm}$ were observed, and the intensity of the peak increased by dipping more membrane coupons in serial order, indicating that unreacted MPD monomers leach out and accumulate in the aqueous rinse solutions. The actual UV absorption values and the concentration of MPD molecules calculated by the UV data were summarized in Table 2. As we initially hypothesized, overall UV intensities observed from the case study 2 were almost 2 3 times higher than those observed from the case study 1 . This result supports that some portion of the released-MPD have immediately reacted with surface-remaining free TMC molecules for the Case Study 1. Moreover, for the polyamide bilayer membranes (HFAPA-on-REFPA) discussed in the previous section, it could be inferred that almost 1 mol $\%$ (for $1^{\text {st }}$ coupon) to the $5 \mathrm{~mol} \%$ of MPD contaminants (for $4^{\text {th }}$ coupon) relative to the total amount of HFAMDA monomers (the concentration of HFAMDA: $0.038 \mathrm{~mol} / \mathrm{L}$ ) 
were present in the $2^{\text {nd }}$ aqueous solution although it is still unclear how much of them were actually involved in the $2^{\text {nd }}$ interfacial reaction.

\subsection{Materials Compositions and Corresponding RO Performance}

The case studies we performed to monitor MPD leaching and its reactivity showed that the material compositions of REFPA could be slightly different depending on its rinsing processes. To understand the structure-property relationship, RO separation performance of the REFPA membranes prepared by Case Study 1 (REFPA rinsed w/ DI water) and Case Study 2 (REFPA rinsed w/Hexane and subsequently w/ DI water) were measured by crossflow filtration of $2000 \mathrm{ppm} \mathrm{NaCl}$ solution and compared with that of polyamide bilayer membranes (HFAPA-on-REFPA) discussed in the previous section (section 3.1. Figure 2). Four sample coupons for each membrane type were evaluated and the performance was averaged for comparison. Figure 5 represents the structures of two REFPA membranes and HFAPA-on-REFPA membranes analogized from their fabrication processes and UV analysis data. The REFPA membrane treated by hexane and water (REFPA_HW) would have a relatively thin layer of aromatic polyamide (Figure 5(a), by case study 2), while the REFPA membrane rinsed with only water (REFPA_W) would have a slightly thicker and/or denser polyamide layer because some of released-MPD molecules in the water directly reacted with surface-remaining free TMC molecules to form additional polyamide bonds (Figure 5(b), by case study 1). Based on the UV study, approximately $6 \times 10^{-5}$ mole of more MPD molecules were consumed to form the additional polyamide bonds on the surface of REFPA_W, compared to REFPA_HW. Since the difference between REFPA_W and REFPA_HW 
was caused by such a trace amount of MPD molecules, it was very difficult to identify any morphological and physical differences between these two membranes by using surface characterization techniques. Both membranes showed a characteristic "peak-andvalley" topographic structure that is typically observed in commercial polyamide membranes with very similar surface roughness as confirmed by AFM (surface roughness: about $60 \mathrm{~nm}$, Supporting Information, Figure S2). Streaming current measurement also showed similar density of surface charges, which originated from surface carboxyate $\left(\mathrm{COO}^{-}\right)$functional groups under neutral $\mathrm{pH}$ conditions (about $-35 \mathrm{mV}$ at $\mathrm{pH}=7$, Supporting Information, Figure S3). However, these two membranes showed a clear difference in the desalination performance, and the REFPA_W membrane showed a higher salt rejection value with similar water flux $(98.5 \pm 0.6 \%, 61 \pm 6 \mathrm{LMH})$ compared to REFPA_HW $(92.8 \pm 2.2 \%, 57 \pm 4 \mathrm{LMH})$. It seems that the additional polyamide bonds densify and/or repair the originally-formed polyamide under-layer, resulting in the enhanced salt rejection. The HFAPA-on-REFPA bilayer membrane prepared by a SIP dipping process is expected to be comprised of a copolyamide top-layer (HFA-polyamide and small portion of REFPA) and the original REFPA under-layer (Figure 5(c)). Since a large portion of surface remaining TMC molecules were reacted with HFAMDA monomers as well as a trace amount of MPD contaminants in the $2^{\text {nd }}$ reaction bath, the HFAPA-on-REFPA membrane showed a relatively lower density of negative charges under neutral conditions (zeta potential: about $-20 \mathrm{mV}$, less $\mathrm{COO}^{-}$groups) compared to two REFPA membranes (Supporting information, Figure S3). The polyamide bilayer membranes showed further improvement in the salt rejection $(99.4 \pm 0.1 \%)$ compared to REF_W, although water flux was slightly decreased. The enhanced salt rejection must be 
attributed to the hydrophobic nature of surface HFA functional groups as we've demonstrated previously [23]. However, to get consistent and reliable desalination performance with the polyamide bilayer membranes, careful control of the SIP process is required, such that the membrane fabrication order doesn't affect the membrane performance. Moreover, it is important to find an optimum composition of the top surface layer to achieve the best combination of salt rejection and water flux.

\subsection{Polyamide Bilayer Membranes: Optimum Material Composition for High RO Performance}

To find an optimum monomers composition for the best desalination performance, polyamide bilayer membranes were fabricated by adding various amounts of MPD into HFAMDA aqueous solutions (mole concentration of HFAMDA: $0.038 \mathrm{~mol} / \mathrm{L}$ ), and by using a frame process in the $2^{\text {nd }}$ reaction step (Figure 6). In the frame process, uncontrollable MPD cross-contamination was eliminated since the $2^{\text {nd }}$ aqueous solution was exposed to only top-surface of a freshly prepared REFPA layer. The molar ratios of MPD additive to the total amount of HFAMDA in the $2^{\text {nd }}$ aqueous solutions were varied from $0 \%$ (pure HFAMDA), $1.2 \%, 2.4 \%, 4.8 \%$, and $100 \%$ (pure MPD). The relative material compositions of the resulting polyamide bilayer membranes were confirmed by FTIR spectroscopic analysis. As shown in Figure 7, all spectra showed characteristic peaks originated from polyamide active layers (amide bonds: 1664 and $1530 \mathrm{~cm}^{-1}$, aromatic rings: $1585,1504,1484$, and $\left.1107 \mathrm{~cm}^{-1}\right)$ and PSF substrates $(1321,1290,1172$, 1150 , and $1245 \mathrm{~cm}^{-1}$ ), and there was no significant difference in these peaks among the samples. However the weak peak at $962 \mathrm{~cm}^{-1}$, which is generated by HFA functional 
groups, gradually decreased toward the polyamide bilayer membranes prepared by adding more MPD additives (Green Boxed Region in Figure 7, (a) (d): $0 \sim 4.8 \mathrm{~mol} \%$ MPD additives), indicating the reduction of HFAMPDA monomer content in the copolyamide top-layers. Finally, the peak at $962 \mathrm{~cm}^{-1}$ disappeared completely when the sample was prepared by using $100 \%$ pure MPD solution (Figure 7(e)).

Figure 8 shows water flux and salt rejection of the resulting polyamide bilayer membranes. It is worthy noting that the membranes for this experiment were prepared by using a new batch of PSF support so that a single-layered REFPA over this new PSF support represented $99.4 \%$ of salt rejection and $44 \mathrm{LMH}$ of water flux. For 0 mol\% MPD addition, pure HFAPA top-layer (0 \% MPD addition) formed on a REFPA underlayer, and the resulting bilayer membrane showed very good desalination performance ( $99.6 \%$ salt rejection and $46 \mathrm{LMH}$ ), which outperforms REFPA. When $1.2 \mathrm{~mol} \%$ of MPD monomer was added into the HFAMDA solution to form the copolyamide top-layer, the salt rejection was even further increased and maximized without adding significant resistance to water transport (99.8\% salt rejection and $45 \mathrm{LMH})$. By adding more than 2.4 mol \% of MPD, however, both water flux and salt rejection gradually decreased. The water flux reduction trend is consistent with the result we have obtained during the crosscontamination study in Section 3.1. Finally, when the top surface layer was formed by pure MPD monomers (100\% MPD), the resulting REFPA-on-REFPA showed very low salt rejection as well as low water flux. Significant reduction in the water flux is likely due to the formation of a much tighter polyamide network on the top surface since monophenyl MPD monomer is more reactive and smaller than bi-phenyl HFAMDA monomer. In general, salt rejection rate is strongly influenced by the surface phenomenon such as an 
interaction between membrane surface and the salts in the feed. The low salt rejection value obtained in the REFPA-on-REFPA indicates that the top REFPA layer (must be amine-rich surface) is not effective enough to repel salts from the membrane surface, and also verifies that HFA functional groups on the surface play an important role to enhance salt rejection behavior. From this study, it is concluded that the 80:1 molar ratio of

HFAMDA and MPD in the $2^{\text {nd }}$ aqueous solution provides the best-performing copolyamide top-layer. Enhanced desalination performance by the copolyamide top-layer seems to be attributed to the combination of the following two factors: (a) hydrophobic nature of HFA functional groups covering the membrane surface and (2) filling and/or repairing any defect sites by the trace amount of small MPD monomer as confirmed by the case studies in Section 3.3 (REFPA_W vs. REFPA_HW).

\section{Conclusions}

The sequential interfacial reaction or polymerization (SIP) is a very straightforward way to modify a conventional polyamide membrane with the aim of improving the membrane's desalination performance, fouling resistance, and/or chlorine resistance. To achieve reliable and consistent performance of the modified membrane, however, basic understanding on the chemistries involved in each reaction process is indeed necessary.

In this study, we have demonstrated the significant impact of cross-contamination in a SIP dipping process while making multiple coupons of polyamide bilayer membranes (HFAPA-on-REFPA) with the same reaction solutions. For the SIP process, unreacted water-soluble MPD monomers carried over from the first interfacial reaction step dissolved and accumulated in the second aqueous solution containing HFAMDA 
monomers, and ultimately participated in the second interfacial reaction to form copolyamide at the top-layer. Depending on the amount of MPD contaminants accumulated in the second aqueous solution, the composition of the resulting copolyamide in the top-layer was varied, resulting in a significant variation of desalination performance (flux reduction and slight increment of salt rejection from $1^{\text {st }}$ sample coupon toward $4^{\text {th }}$ sample coupon).

MPD leaching in the $2^{\text {nd }}$ aqueous solution and the subsequent reaction of the released MPD with surface-remaining TMC molecules were clearly confirmed by two case studies conduced with UV spectroscopic analysis (Case 1: REFPA $\rightarrow$ dipping into DI water $\rightarrow$ UV analysis, and Case 2: REFPA $\rightarrow$ rinsing with hexane $\rightarrow$ dipping into DI water $\rightarrow$ UV analysis). These case studies indicated that the material compositions of REFPA could be slightly different depending on its rinsing processes as well. REFPA_W prepared by Case Study 1 must have additional polyamide bonds when compared to REFPA_HW prepared by Case Study 2 due to the direct reaction of the released-MPD and surface-remaining TMC molecules during the water rinse process. Performance comparison among REFPA_HW, REFPA_W, and HFAPA-on-REFPA (by SIP dipping process) supported that a trace amount of MPD involvement in the $2^{\text {nd }}$ interfacial reaction might be necessary to improve overall desalination performance (salt rejection: $92.8 \%$ for REFPA_HW < 98.5\% for REFPA_W) and also verified that HFA functional groups covering the membrane top surface play an important role in the enhancement of RO separation behavior (salt rejection: $99.4 \%$ for HFAPA-on-REFPA).

Through in-depth evaluation on the performance of top surface layers, which were prepared by adding various known-amount of MPD into $2^{\text {nd }}$ HFAMDA solution 
(copolymerization) and also by applying a frame process (no cross-contamination issue), we have successfully identified an ideal composition (molar ratio of 80:1) between HFAMDA and MDA to achieve the best RO desalination performance $(99.8 \%$ salt rejection, $45 \mathrm{LMH}$ water flux).

This study clearly addresses an important factor that should be considered during sequential interfacial polymerization to obtain a reliable and high performing coating layer on a conventional polyamide membrane. The $2^{\text {nd }}$ layer-copolymerization approach (trace amount of MPD addition to the HFAMDA solution), which we have performed to optimize RO separation behaviors of polyamide bilayer membranes, could be also applied to other material systems (ex. anti-fouling materials) to improve overall desalination performance.

\section{Acknowledgements}

This work was performed under a joint development agreement between IBM Research and the King Abdulaziz City for Science \& Technology (KACST).

\section{References}

[1] R.F. Service, Desalination Freshens Up, Science (Washington, DC, United States), 313 (2006) 1088-1090.

[2] M.A. Shannon, P.W. Bohn, M. Elimelech, J.G. Georgiadis, B.J. Marinas, A.M. Mayes, Science and technology for water purification in the coming decades, Nature (London, United Kingdom), 452 (2008) 301-310.

[3] D.G. Cahill, V. Freger, S.-Y. Kwak, Microscopy and microanalysis of reverseosmosis and nanofiltration membranes, MRS Bulletin, 33 (2008) 27-32.

[4] T. Matsuura, Progress in membrane science and technology for seawater desalination - a review, Desalination, 134 (2001) 47-54. 
[5] R.J. Petersen, Composite reverse osmosis and nanofiltration membranes, Journal of Membrane Science, 83 (1993) 81-150.

[6] J. Benavente, J.M. Garcia, J.G. de la Campa, J. de Abajo, Determination of some electrical parameters for two novel aliphatic-aromatic polyamide membranes, Journal of Membrane Science, 114 (1996) 51-57.

[7] J.E. Cadotte, Reverse osmosis membrane, in, (Midwest Research Institute, USA). Application: US, 1981, pp. 9 pp Cont -in-part of U S Ser No 958,593.

[8] Y.-H. La, R. Sooriyakumaran, D.C. Miller, M. Fujiwara, Y. Terui, K. Yamanaka, B.D. McCloskey, B.D. Freeman, R.D. Allen, Novel thin film composite membrane containing ionizable hydrophobes: $\mathrm{pH}$-dependent reverse osmosis behavior and improved chlorine resistance, Journal of Materials Chemistry, 20 (2010) 4615-4620.

[9] X. Wang, T.-M. Yeh, Z. Wang, R. Yang, R. Wang, H. Ma, B.S. Hsiao, B. Chu, Nanofiltration membranes prepared by interfacial polymerization on thin-film nanofibrous composite scaffold, Polymer, 55 (2014) 1358-1366.

[10] L. Yung, H. Ma, X. Wang, K. Yoon, R. Wang, B.S. Hsiao, B. Chu, Fabrication of thin-film nanofibrous composite membranes by interfacial polymerization using ionic liquids as additives, Journal of Membrane Science, 365 (2010) 52-58.

[11] R.L. Riley, R.L. Fox, C.R. Lyons, C.E. Milstead, M.W. Seroy, M. Tagami, Spiralwound poly(ether/amide) thin-film composite membrane systems, Desalination, 19 (1976) 113-126.

[12] S.D. Arthur, Preparation and uses of multilayer reverse osmosis membrane of polyamide-urea, in, (du Pont de Nemours, E. I., and Co., USA). Application: US, 1991, pp. 7 pp.

[13] L.-F. Liu, S.-C. Yu, Y. Zhou, C.-J. Gao, Study on a novel polyamide-urea reverse osmosis composite membrane (ICIC-MPD), Journal of Membrane Science, 281 (2006) $88-94$.

[14] S.G. Kim, D.H. Hyeon, J.H. Chun, B.-H. Chun, S.H. Kim, Nanocomposite poly(arylene ether sulfone) reverse osmosis membrane containing functional zeolite nanoparticles for seawater desalination, Journal of Membrane Science, 443 (2013) 10-18.

[15] K.P. Lee, T.C. Arnot, D. Mattia, A review of reverse osmosis membrane materials for desalination. Development to date and future potential, Journal of Membrane Science, 370 (2011) 1-22.

[16] D. Rana, T. Matsuura, Surface Modifications for Antifouling Membranes, Chemical Reviews (Washington, DC, United States), 110 (2010) 2448-2471. 
[17] T. Knoell, Municipal wastewater. Chlorine's impact on the performance and properties of polyamide membranes, Ultrapure Water, 23 (2006) 24-31.

[18] S.H. Kim, S.-Y. Kwak, B.-H. Sohn, T.H. Park, Design of TiO2 nanoparticle selfassembled aromatic polyamide thin-film-composite (TFC) membrane as an approach to solve biofouling problem, Journal of Membrane Science, 211 (2003) 157-165.

[19] W. Choi, J. Choi, J. Bang, J.-H. Lee, Layer-by-Layer Assembly of Graphene Oxide Nanosheets on Polyamide Membranes for Durable Reverse-Osmosis Applications, ACS Appl. Mater. Interfaces, 5 (2013) 12510-12519.

[20] Y. Zhou, S. Yu, C. Gao, X. Feng, Surface modification of thin film composite polyamide membranes by electrostatic self deposition of polycations for improved fouling resistance, Separation and Purification Technology, 66 (2009) 287-294.

[21] W.E. Mickols, Composite polyamide-polyoxyalkylene graft polymers as reverse osmosis and nanofiltration membranes, in, The Dow Chemical Company, USA . 2000, pp. $20 \mathrm{pp}$.

[22] J. Xu, Z. Wang, L. Yu, J. Wang, S. Wang, Reverse osmosis membrane with regenerable anti-biofouling and chlorine resistant properties, Journal of Membrane Science, 435 (2013) 80-91.

[23] Y.-H. La, J. Diep, R. Al-Rasheed, D. Miller, L. Krupp, G.M. Geise, A. Vora, B. Davis, M. Nassar, B.D. Freeman, M. McNeil, G. Dubois, Enhanced desalination performance of polyamide bi-layer membranes prepared by sequential interfacial polymerization, Journal of Membrane Science, 437 (2013) 33-39.

[24] G. Kang, M. Liu, B. Lin, Y. Cao, Q. Yuan, A novel method of surface modification on thin-film composite reverse osmosis membrane by grafting poly(ethylene glycol), Polymer, 48 (2007) 1165-1170.

[25] H. Zou, Y. Jin, J. Yang, H. Dai, X. Yu, J. Xu, Synthesis and characterization of thin film composite reverse osmosis membranes via novel interfacial polymerization approach, Separation and Purification Technology, 72 (2010) 256-262. 


\section{Figures}

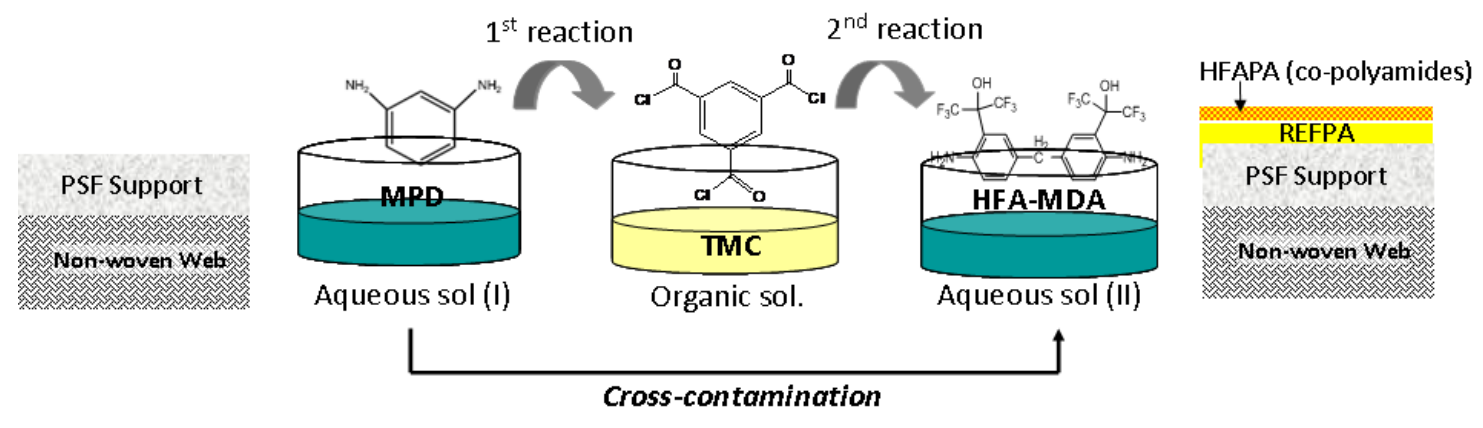

Figure 1. A schematic drawing showing a cross-contamination issue in the sequential interfacial polymerization (SIP) process based on the subsequent membrane dipping. 

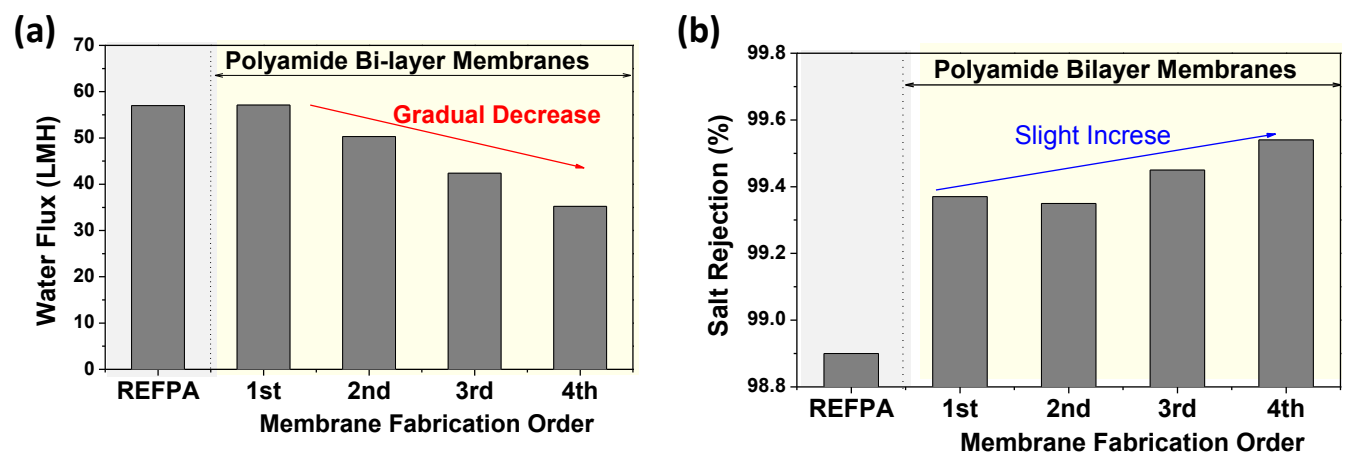

Figure 2. Water flux (a) and salt rejection (b) of REFPA and four coupons of polyamide bilayer membranes $\left(1^{\text {st }} \sim 4^{\text {th }}\right)$ prepared by sequential interfacial polymerization in the manner of serial membrane dipping. 
(a) Case Study 1: REFPA_standard

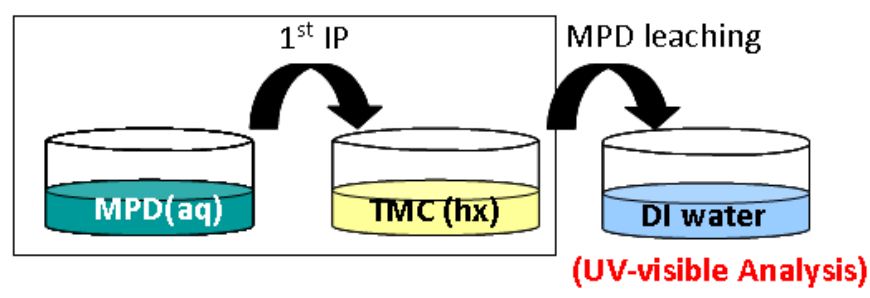

(b) Case Study 2: REFPA_w/hexane rinse

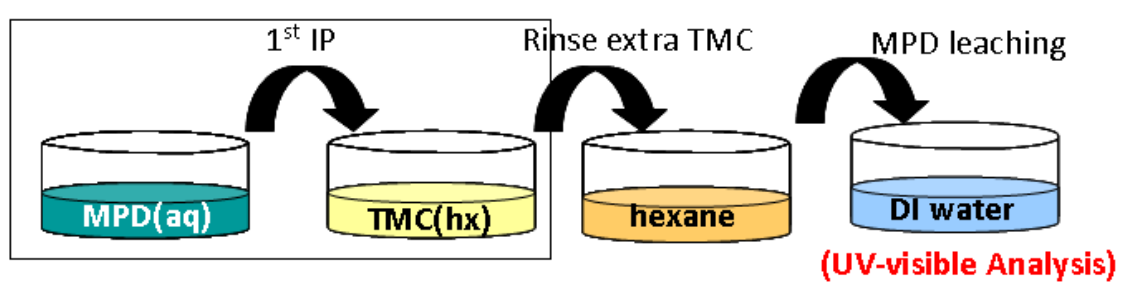

Figure 3. Schemes for the case studies to prove MPD leaching and its reactivity in the $2^{\text {nd }}$ aqueous solution 

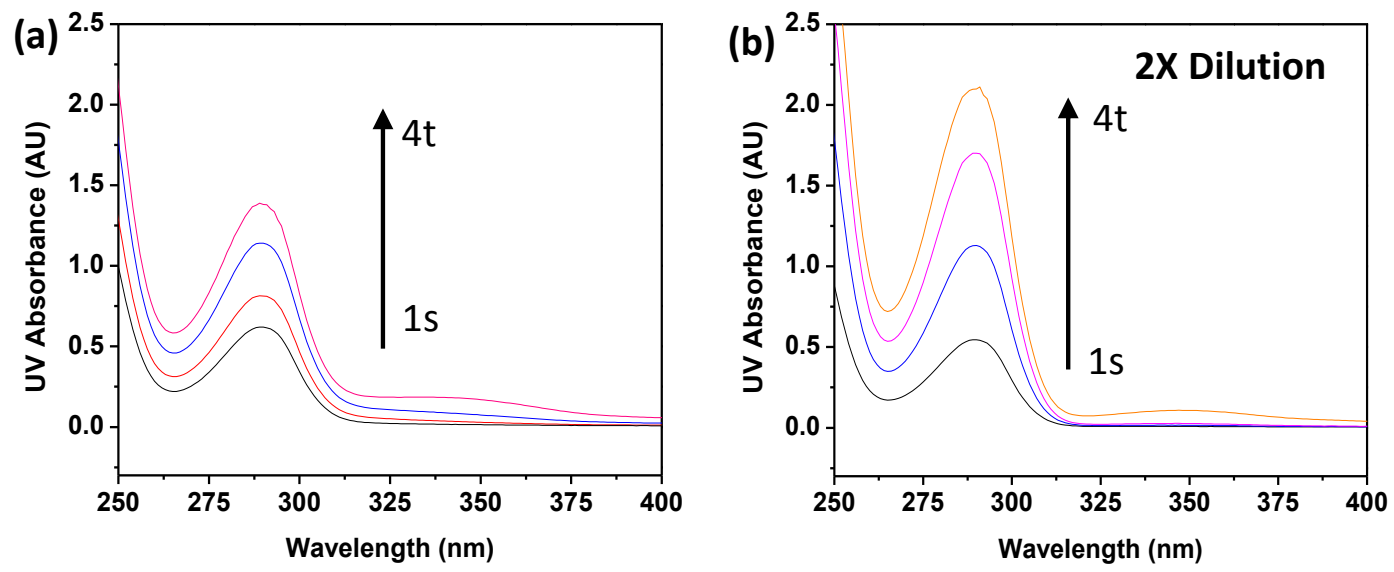

Figure 4. Plots showing UV absorption intensities of water samples collected from (a) case study 1 and (b) case study 2 . The water samples for case study 2 were diluted two times to avoid saturation of UV signals. 
(a) REFPA_HW (Case Study 2)

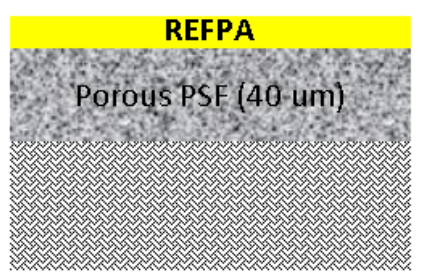

Salt Rejection: $\quad 92.8 \pm 2.2 \%$ Water Flux: (b) REFPA_W (Case Study 1)

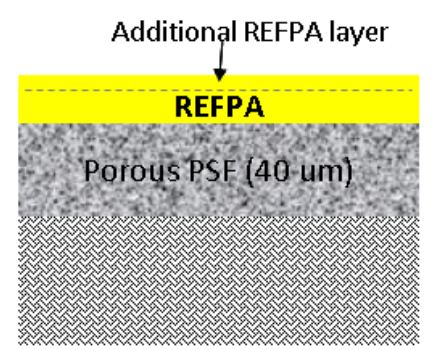

$98.5 \pm 0.6 \%$

$61 \pm 6 \mathrm{LMH}$ (c) HFAPA-On-REFPA

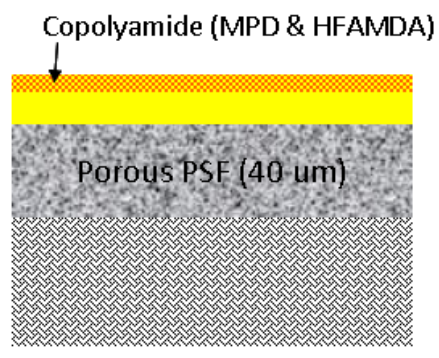

$99.4 \pm 0.1 \%$ $46 \pm 10$ LMH

Figure 5. Expected structures of RO membranes made by three different processes and their desalination performance (a) REFPA made by the process shown in the case study 2 (w/additional hexane rinse), (b) REFPA made by the process shown in the case study 1 , and (c) polyamide bilayer membrane made by the SIP process shown in Figure 1. 


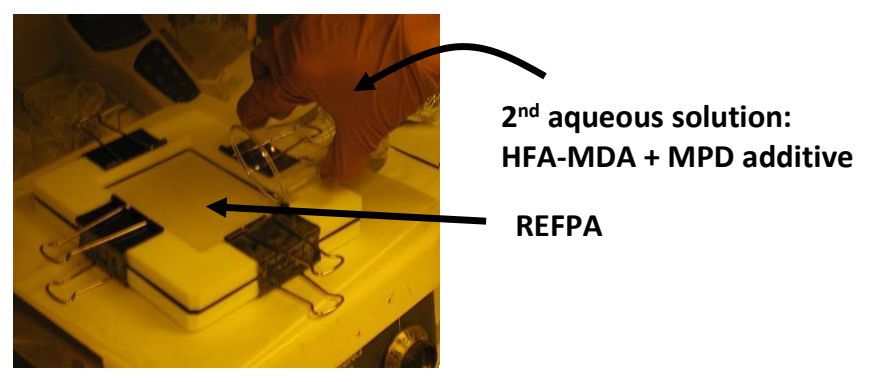

Figure 6. A photograph showing a frame process to form polyamide bilayer membranes featuring HFAPA top-layer (copolymerization of HFAMDA and MPD additive) on a REFPA under-layer without cross-contamination 


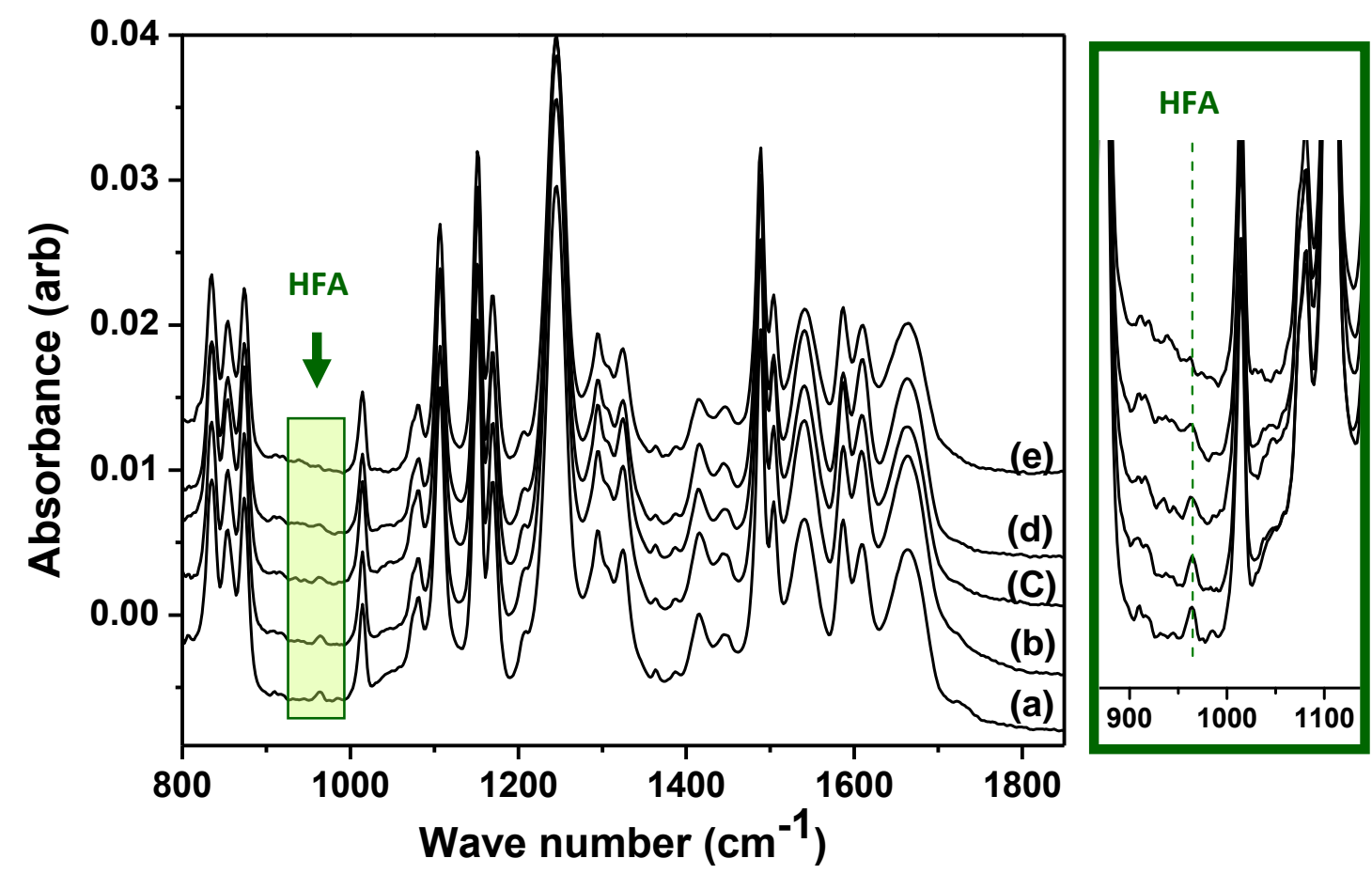

Figure 7: FTIR spectra of polyamide bilayer membranes prepared by adding various amount of MPD additive into the 2nd aqueous solution containing HFA-MDA monomer ((a) $0 \%$, (b) $1.2 \%$, (c) $2.4 \%$, (d) $4.8 \%$, and (e) $100 \%$ MPD additions). (a), (b) (d), and (e) spectra were originated from a pure HFAPA top-layer, copolyamide top-layers, and a pure REFPA top-layer on REFPA under-layers, respectively. Green box on the right: magnified spectra in the range of $900 \sim 1100 \mathrm{~cm}^{-1}$. 

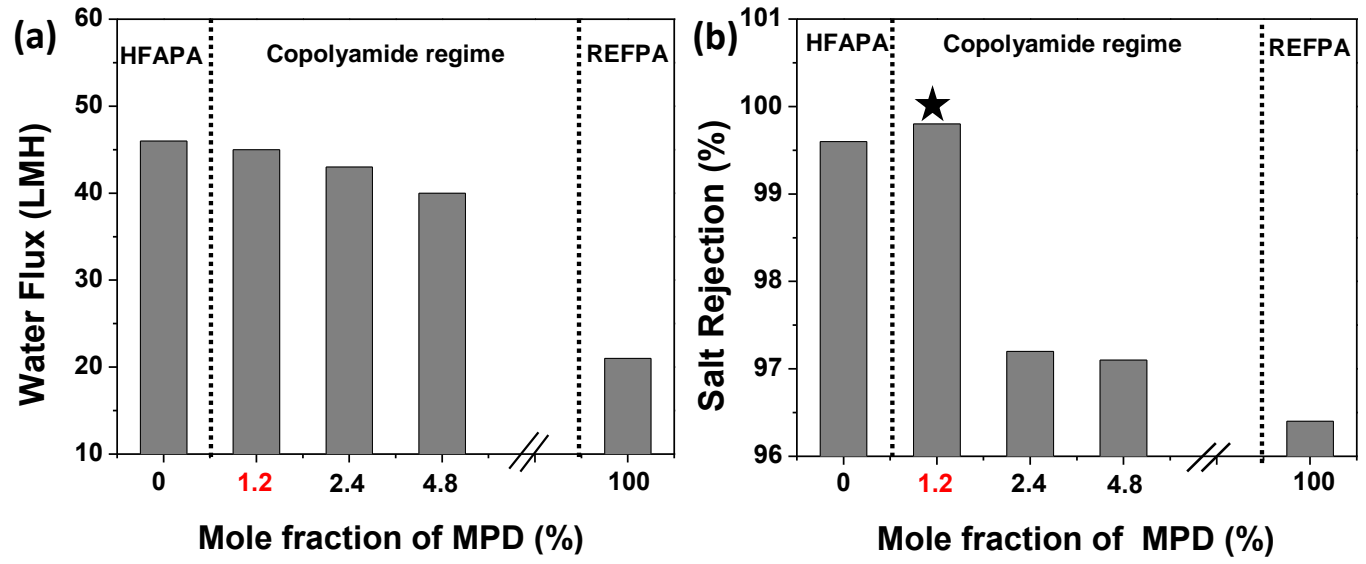

Figure 8. (a) Water flux and (b) salt rejection of polyamide bilayer membranes prepared by adding MPD additive into the 2 nd aqueous solution containing base HFA-MDA monomer. The mole fractions of MPD additive were varied from $0 \%$ to $100 \%$ to the total amount of HFAMDA monomer. 0\% MPD addition, $1.2 \sim 4.8 \%$ MPD additions, and $100 \%$ MPD addition result in pure HFAPA top-layer, copolyamide top layer, and pure REFPA top layer on a REFPA under-layer, respectively. Data were acquired by crossflow filtration of $2000 \mathrm{ppm} \mathrm{NaCl}$ solution at 400 psi. 


\section{Tables}

\begin{tabular}{|c|c|c|c|}
\hline \multirow{2}{*}{$\begin{array}{c}\text { HFAPA-on-REFPA } \\
\text { (fabrication order) }\end{array}$} & \multicolumn{2}{|c|}{$\begin{array}{c}\text { XPS intensity and } \\
\text { atomic ratio }\end{array}$} & \multirow{2}{*}{$\begin{array}{c}\text { Water contact } \\
\text { angle ( }\end{array}$} \\
\cline { 2 - 4 } & $\mathrm{F}(1 \mathrm{~s})$ & $\mathrm{F} / \mathrm{C}$ & \\
\hline 1st & 17.1 & 0.27 & $133.1 \pm 1.3$ \\
\hline 2nd & 15.3 & 0.24 & $129.4 \pm 1.8$ \\
\hline 3rd & 13.3 & 0.21 & $128.8 \pm 4.6$ \\
\hline 4th & 12.8 & 0.20 & $118.9 \pm 4.9$ \\
\hline
\end{tabular}

Table 1. XPS F(1s) concentration, atomic ratio (F/C), and water contact angle of the layered polyamide membranes fabricated by the SIP dipping process in serial order with the same solution baths. 


\begin{tabular}{|c|c|c|c|c|}
\hline & \multicolumn{2}{|c|}{ UV intensity } & \multicolumn{2}{c|}{$\begin{array}{c}\text { Accumulated MPD } \\
\text { concentration (mole/L) }\end{array}$} \\
\hline & Case 1 & Case 2 & Case 1 & Case 2 \\
\hline 1st Coupon & 0.62 & 1.09 & $2.7 \times 10-4$ & $4.8 \times 10-4$ \\
\hline 2nd Coupon & 0.82 & 2.26 & $3.6 \times 10-4$ & $10 \times 10-4$ \\
\hline 3rd Coupon & 1.14 & 3.40 & $5.0 \times 10-4$ & $15 \times 10-4$ \\
\hline 4th Coupon & 1.39 & 4.22 & $6.1 \times 10-4$ & $19 \times 10-4$ \\
\hline
\end{tabular}

Table 2. Summary of UV absorption values and accumulated MPD concentrations obtained from case studies 1 and 2. The actual numbers of MPD molecules released from each sample coupon (4" x 5 ") into a $200 \mathrm{~mL}$ aqueous solution were approximately $3 \mathrm{x}$ $10^{-5}$ mole for the Case 1 and $9 \times 10^{-5}$ mole for the Case 2 


\section{Supporting Information}
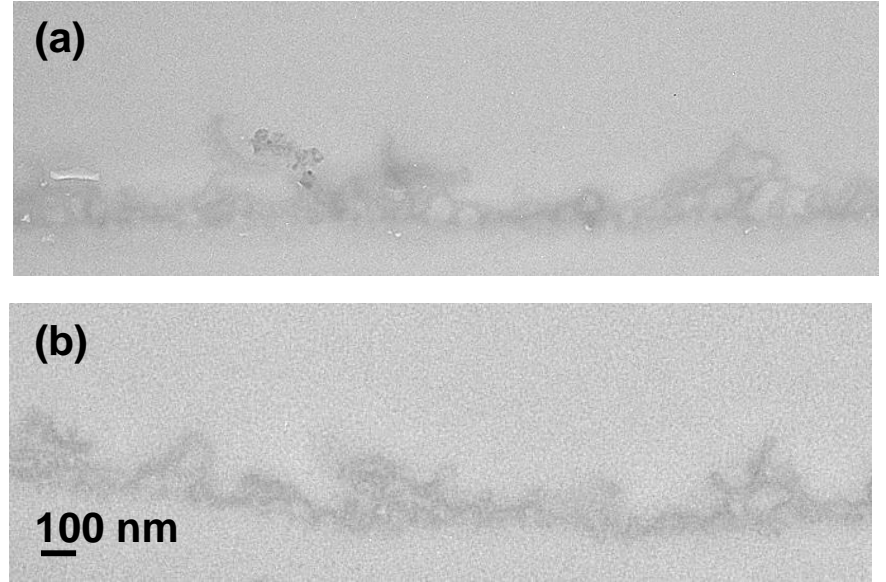

Figure S1. Cross-sectional TEM images of (a) REFPA and (b) HFAPA-on-REFPA membranes. The first sample coupon of HFAPA-on-REFPA was used for TEM analysis. 

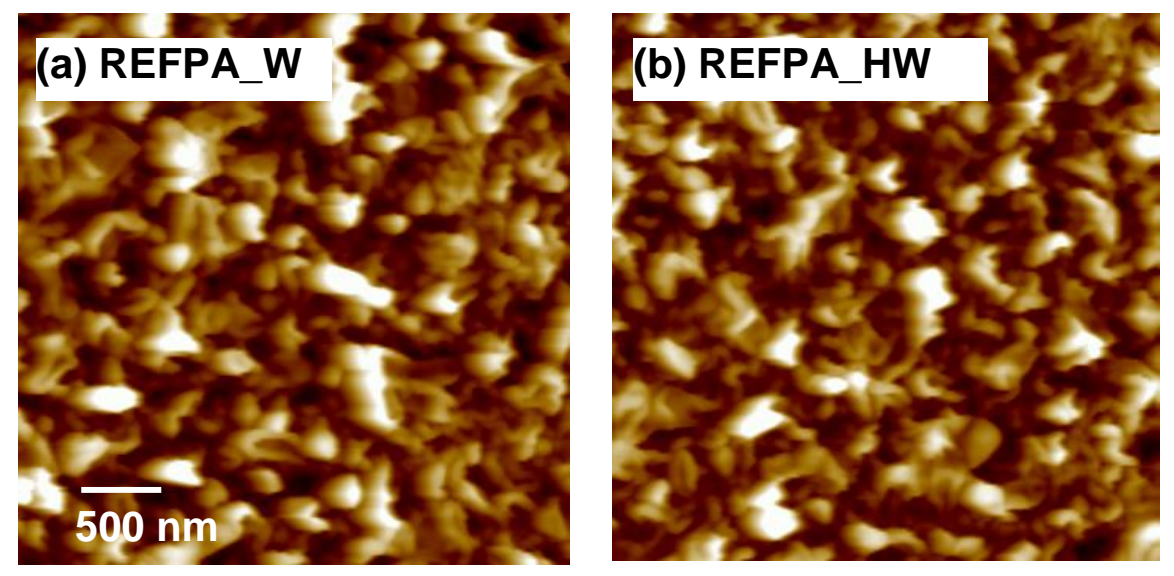

Figure S2: AFM topographic images of (a) REFPA_W and (b) REFPA_HW; Surface roughness values for REFPA_W and REFPA_HW are $61 \pm 3 \mathrm{~nm}$ and $60 \pm 4 \mathrm{~nm}$, respectively. 


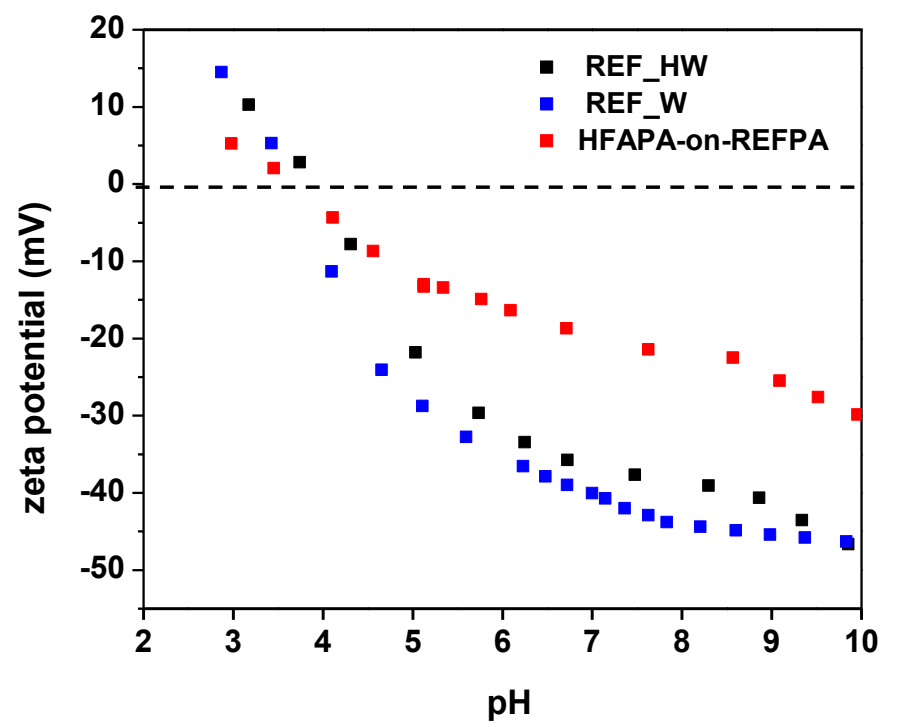

Figure S3: pH-dependant zeta-potential curves of REF_W (blue, prepared by Case Study 1), REF_HW (Black, prepared by Case Study 2), and HFAPA-on-REFPA (Red, prepared by the scheme in Figure 2). All experiments were performed in a millimolar $\mathrm{KCl}$ solution 